\title{
Alternative Axiomatizations of the Conditional System VC
}

\author{
Claudio E. A. Pizzi*
}

Received: 17 September 2018 / Accepted: 17 May 2019

Abstract: The central result of the paper is an alternative axiomatization of the conditional system VC which does not make use of Conditional Modus Ponens: $(A>B) \supset(A \supset B)$ and of the axiom-schema CS: $(A \wedge B) \supset(A>B)$. Essential use is made of two schemata, i.e. X1: $(A \wedge \diamond A) \supset(\diamond A><A)$ and $\mathrm{T}: \square A \supset A$, which are subjoined to a basic principle named Int: $(A \wedge B) \supset(\diamond A>\diamond B)$. A hierarchy of extensions of the basic system V called VInt, VInt1, VInt1T is then construed and submitted to a semantic analysis. In Section 3 VInt1T is shown to be deductively equivalent to VC. Section 4 shows that in VC the thesis $\mathrm{X} 1$ is equivalent to $\mathrm{X} 1 \vee:(\diamond A><A) \vee(\diamond \neg A><\neg A)$, so that VC is also equivalent to a variant of VInt1T here called VInt1T $^{o}$. In Section 6 both X1 and X1V offer the basis for a discussion on systems containing CS, in which it is argued that they cannot avoid various kinds of partial or full trivialization of some non truth-functional operators.

Keywords: Conditional logic; centering condition; trivialization; modal collapse.

\footnotetext{
*Emeritus, University of Siena

Dipartimento di Scienze Sociali, Politiche e Cognitive, University of Siena, via Roma 56, 53100 Siena, Italy

凶izizi4@gmail.com
}

(c) The Author. Journal compilation (c) The Editorial Board, Organon F. 


\section{The conditional systems V, VW, VC and C2}

The system of conditional logic named VC in (Lewis 1973) (but named C1 in Lewis 1971) is currently considered the central system inside the family of so-called "classical conditional logics". (Lewis 1973, 130) writes: "In my analysis of counterfactuals, I officially imposed centering and none of the other conditions, VC is therefore my official logic for the counterfactual interpretation". However, Lewis was not fully satisfied with the various axiomatizations he found for this system: for this reason he was inclined to prefer an axiomatization whose primitive notion was that of comparative possibility, here represented by the symbol $\prec$.

The axiomatization which we will use as a starting point may be found in (Nute 1980). In what follows $A, B, C, \ldots$ will be (meta)variables for wffs, $\neg$, $\supset, \wedge, \vee$ and $\equiv$ will be symbols for the standard truth-functional operators, while the symbol $>$ will be used for the primitive conditional operator, $><$ for conditional equivalence and $\ni$ for the dual of $>$. The relation symbolized by $A \ni B$ will be read as "A is cotenable with $\mathrm{B}$ ". The formation rules are standard. Parentheses will be omitted when no ambiguity arises around wffs having $>$, $\ni$ or $><$ as the main operator.

The definitions of the auxiliary operators are:

Def $><A><B={ }_{d f} A>B \wedge B>A$

Def $\ni \quad A \ni B={ }_{d f} \neg(A>\neg B)$;

Def $\square \quad \square A=_{d f} \neg A>A$;

Def $\diamond \quad \diamond A={ }_{d f} \neg(A>\neg A)$;

Def $\neg \quad A \multimap B={ }_{d f} \square(A \supset B)$.

The relation of comparative possibility $A \prec B$ (which will not be used in the present paper) is defined by Lewis as $\diamond A \wedge((A \vee B)>(A \wedge \neg B))$, while $A \preccurlyeq B$ stands for $\neg(B \prec A)$.

The axiom schemata of VC, according to (Nute 1980, 129), may be formulated as follows:

PC All the tautologies of the truth-functional propositional calculus;

ID $A>A$;

MOD $\neg A>A \supset B>A$; 
CSO $A><B \supset(A>C \equiv B>C)$;

$\mathrm{CV} \quad(A>B \wedge A \ni C) \supset(A \wedge C)>B$

$\mathrm{CMP} A>B \supset(A \supset B)$;

CS $\quad(A \wedge B) \supset A>B$.

The rules are:

MP $\quad$ From $\vdash A$ and $\vdash A \supset B$ to infer $\vdash B$;

RCEC From $\vdash A \equiv B$ to infer $\vdash C>A \equiv C>B$;

RCK From $\vdash\left(A_{1} \wedge \ldots \wedge A_{n}\right) \supset B$ to infer $\vdash\left(C>A_{1} \wedge \ldots \wedge C>A_{n}\right) \supset C>B$, for $n \geq 0$.

The following rule RCE is derivable as a special case of RCK:

RCE From $\vdash A \supset B$ to infer $\vdash A>B$.

The axiom schema CSO expresses a particular feature of any conditional logic which contains it: the possibility of replacing $><$-equivalents in the antecedent of a conditional. The rule

RCEA From $\vdash A \equiv B$ to infer $\vdash A>C \equiv B>C$

follows from CSO and RCE, since RCE yields the derived rule

RCEq From $\vdash A \equiv B$ to infer $\vdash A><B$.

It follows that replacement of proved $\equiv$-equivalents is a universally valid rule in VC. In what follows we shall call $a$-equivalents any two statements which can be reciprocally replaced in the antecedent of a conditional via an application of CSO or of RCEq. The subsystem of VC which results from removing axiom CS is called VW. Removing CMP (the so-called Conditional Modus Ponens) from VW the resulting minimal system is called $\mathbf{V}$. Any conditional system which has among its rules RCEC and RCEA is called classical in (Chellas 1975), so V and all its extensions are classical conditional logics.

Lewis showed that the fragment of $\mathbf{V W}$ and $\mathbf{V C}$ containing only $\square$ and truth-functional operators is exactly $\mathbf{K T}$, while the modal fragment of $\mathbf{V}$ is the minimal system $\mathbf{K}$. The collapse-formula (Ban) $A \supset \square A$ is underivable in all extensions of VC written in the >-language considered in (Lewis 1973), so 
none of these systems is trivial. ${ }^{1}$ If $\top$ stands for $A \vee \neg A$ and $\perp$ for $\neg \top$, the collapse is however derivable in $\mathbf{V C}$ for a second modal operator $\square$ defined by

\section{$\operatorname{Def} \unrhd \square A=_{d f} \top>A$.}

The collapse of $\square$ is a consequence of axiom CS. In fact, putting $T$ in place of $A$ in CS the result is $(\top \wedge B) \supset \top>B$, so $B \supset \square B$. This formula will be called Ban $\square$. The stronger equivalence $B \equiv \square B$ will be called Triv $\square$ and is also derivable in $\mathbf{V C}$, given that $\top>B$ (i.e. $\square B$ ) implies $\top \supset B$ by CMP, hence $B$ by $\mathbf{P C}$.

Various versions of the model theory for conditional language have been proposed in the literature (see for instance Lewis 1971). For our aims the semantics for conditional systems may be outlined as follows. A V-model is a 4-ple $\langle W, f, R, V\rangle$, where:

(i) $W$ is a non-empty set of possible worlds $\{a, b, c, \ldots\}$,

(ii) $f$ is a function from couples of propositions and worlds to sets of worlds such that, for every proposition $A$ and world $i, f(A, i)$ is a subset of $W$ which is also a subset of $A$-worlds, i.e. of worlds where $A$ is true (intuitively, the set of $A$-worlds "more similar" to $i$ ). The properties of $f$ are the basic properties of so-called selection functions, as formulated by (Stalnaker 1968), with the essential difference that the selection function $f$ here has sets of worlds as its values (set-selection functions) while Stalnaker asks that $f$ selects a unique world. ${ }^{2}$

(iii) $R$ is a binary relation on $W$;

(iv) if $j$ belongs to $f(A, i)$ then $i R j$;

(v) $V$ is an evaluation function that is defined in a standard way as far as

${ }^{1}$ In (Lewis 1973, 121), a trivial system named CA is formulated in a language containing the symbol $\prec$. The trivializing schema is $A \prec B \equiv A \supset B$.

${ }^{2}$ (Lewis 1971, 75) proposes the following minimal clauses for the definition of (non-centered) set-selection functions, where $A$ is a sentence and $[A]$ the set of $A$-worlds:

(a) $f(A, i) \subseteq[A]$ (which means that $V(A, j)=1$ at every $j$ in $f(A, i)$ );

(b) if $f(A, i) \subseteq[B]$ and $f(B, i) \subseteq[A]$, then $f(A, i)=f(B, i)$;

(c) either $f(A \vee B, i) \subseteq[A]$ or $f(A \vee B, i) \subseteq[B]$ or $f(A \vee B, i)=f(A, i) \cup f(B, i)$.

We are assuming in what follows that the function $f$ has the properties stated in (a), (b), (c). 
truth-functional operators are concerned and that w.r.t. the >-operator satisfies the following clause:

(vi) $V(A>B, i)=1$ iff $V(B, j)=1$ at every $j$ in $f(A, i)$ (this means that if $f(A, i)$ is $\emptyset$ the right part of the equivalence (vi) is vacuously satisfied: so, in this special case, $V(A>B, i)=1$ for every $B$, so $V(A>\neg A, i)=V(\square \neg A, i)=1$. On the other hand, if $\square \neg A$ is true at $i, f(A, i)$ is $\emptyset$; otherwise, if $f(A, i) \neq \emptyset$, by clause (iv) some $j$ exists such that $i R j$, so $\diamond A$ is true at $i)$;

A VW-model has all the properties (i)-(vi) and furthermore the following:

- (Weak Centering) if $V(A, i)=1$ then $i \in f(A, i)$.

Weak Centering validates CMP, so its instantiation $\neg A>A \supset(\neg A \supset A)$, so $\square A \supset A$. Then in $\mathbf{V W}$-models the relation $R$ has the property of being reflexive.

A VC-model has all the properties of $\mathbf{V W}$-models and also the following:

- (Centering) If $V(A, i)=1$, then $f(A, i)=\{i\}$

which validates axiom CS. Stalnaker's system C2 has in place of CS the so called "Conditional Excluded Middle" (CEM):

$\mathrm{CEM} \quad A>B \vee A>\neg B$.

C2-models are as VC-models with the only difference that the set $f(A, i)$ is a singleton. As proved by (Lewis 1973), V, VW, VC, C2 are sound and complete for the above defined semantics.

\section{The conditional systems VInt, VInt1 and VInt1T}

We remark that all the schemata used in the above introduced axiomatization of VC, with the only exception of Id, are schemata involving two metavariables. In what follows we will consider three schemata, two of which contain a single metavariable, which can be used to provide an alternative axiomatization of $\mathbf{V C}$ :

Organon F 26 (3) 2019: 427-445 


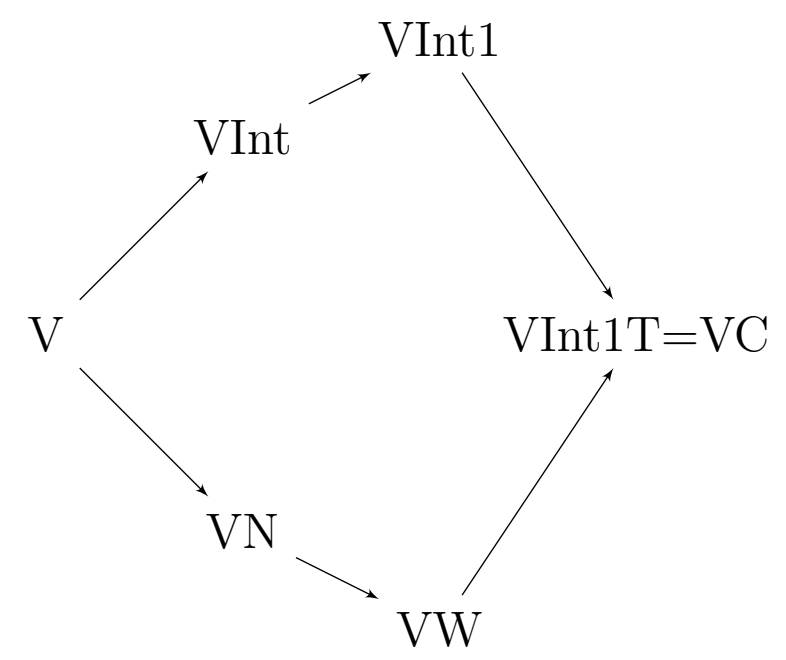

Figure 1: Converging extensions of $\mathbf{V}$

Int $(A \wedge B) \supset \diamond A>\diamond B$

$\mathrm{X} 1 \quad(A \wedge \diamond A) \supset \diamond A><A$;

$\mathrm{T} \square A \supset A$.

A wff which is equivalent to Int is the schema:

Int+ $(A \wedge B) \supset \diamond A><\diamond B$.

The diagram in Figure 1 offers a visualization of the inclusion relation (represented by the arrow) among six conditional systems with the following names:

$$
\begin{array}{ll}
\text { VInt } & =\mathbf{V}+\mathbf{I n t} ; \\
\text { VInt1 } & =\mathbf{V}+\text { Int }+\mathrm{X} 1 ; \\
\text { VInt1T } & =\mathbf{V}+\mathrm{Int}+\mathrm{X} 1+\mathrm{T} ; \\
\mathbf{V N} & =\mathbf{V}+\mathrm{N}(\mathrm{N}: \diamond \top) ;^{3} \\
\mathbf{V W} & =\mathbf{V}+\mathrm{CMP} ; \\
\mathbf{V C} & =\mathbf{V}+\mathrm{CMP}+\mathrm{CS} .
\end{array}
$$

The right side of the diagram in Figure 1 is a fragment of the map of conditional logics reproduced in Fig. 5 of (Lewis 1973, 131). Other combinations of schemata (for instance $\mathbf{V}+\mathrm{X} 1$ ) will not be taken here in consideration for sake of simplicity. In what follows we prove that the systems in the lower side 
of the diagram are distinct systems, or more exactly that each one is properly included in the next of the sequence. In other words we have to prove that Int is not a V-theorem, $\mathrm{X} 1$ is not a VInt-theorem and that $\mathrm{T}$ is not a VInt1theorem. The first result is simple since we have at our disposal a soundness and completeness theorem for $\mathbf{V}$ w.r.t. the class of $\mathbf{V}$-models.

\section{Proposition 2.1. Int is not a $\boldsymbol{V}$-theorem.}

Proof. It is enough to prove that Int, i.e. $(A \wedge B) \supset \diamond A>\diamond B$, is falsified in a $\mathbf{V}$-model with the following consistent properties: $W=\{i, j\}$ and for any formulas $A$ and $B, j \in f(\diamond A, i), V(A, i)=V(B, i)=1, V(B, j)=0, j R j$. In this model then $V(\diamond B, j)=0$. Since $V(\diamond A, j)=1$ for the properties of $f$ (see footnote 2, clause a) $V(\diamond A>\diamond B, i)=0$. Given that $V(A \wedge B, i)=1$, Int has value 0 at $i$. Since all $\mathbf{V}$-theorems are valid in all $\mathbf{V}$-models for the soundness theorem, this amounts to a disproof of Int in $\mathbf{V}$.

In order to reach the other expected results we associate a semantics to the relevant systems in this way. First we define two properties of $\mathbf{V}$-models which we call f-Symmetry and Pseudo-centering.

$(f S) \quad$ For every $i$ in $W$ and for every $A$ such that $V(A, i)=1$, if $j \in$ $f(\diamond A, i)$, then $i \in f(\diamond A, j)$.

$(P s C)$ For every $i$ in $W$, and for every $A$ such that $V(A, i)=1$ then, for every $j$ in $f(A, i), f(A, j)=\{j\}$.

A consequence of $(f S)$ is:

$\left(f S^{o}\right)$ For every $i$ in $W$ and for every $A$ such that $V(A, i)=1$, if $j \in$ $f(\diamond A, i)$, then $i R j$ and $j R i$.

A consequence of $(P s C)$ is:

$\left(P s C^{o}\right)$ For every $i$ in $W$, and for every $A$ such that $V(A, i)=1$ then, for every $j$ in $f(A, i), j R j$.

We prove:

Proposition 2.2. VInt is sound w.r.t. the class of $f$-symmetrical $\boldsymbol{V}$-models.

Proof. Suppose that Int has value 0 at some $i$ of some $\mathbf{V}$-model $M$ which is also $f$-symmetrical. Then $V(A, i)=V(B, i)=1$ and $V(\diamond A>\diamond B, i)=0$. 
This means that there is a $j$ belonging to $f(\diamond A, i)$ such that $V(\diamond A, j)=1$ and $V(\diamond B, j)=0$. But due to the property of $f$-symmetry, $i \in f(\diamond A, j)$. By the properties of $\mathbf{V}$-models, $\left(f S^{o}\right)$ implies that $j R i$. So, given that $V(\diamond B, j)=0$, $B$ has value 0 at all worlds $k$ such that $j R k$ : thus, given that $j R i, V(B, i)=0$ at $i$, contrary to the supposition that $V(B, i)=1$.

One may check by induction on the length of proofs that all VInt-axioms schemata are valid for this semantics and that the rules preserve validity, so every VInt-thesis is valid in all $\mathbf{V}$-models which are $f$-symmetrical.

Proposition 2.3. VInt1 is sound w.r.t. the class of all $f$-symmetrical and pseudo-centered $\boldsymbol{V}$ - models.

Proof. In Proposition 2.2 it is proved that Int is valid in the class of $f$ symmetrical $\mathbf{V}$-models, so it is a fortiori valid in the class of pseudo-centered and $f$-symmetrical models. Since $\mathrm{X} 1$ is $(A \wedge \diamond A) \supset \diamond A><A$, it is equivalent to $((A \wedge \diamond A) \supset(A>\diamond A)) \wedge((A \wedge \diamond A) \supset(\diamond A>A))$.

First suppose that $(A \wedge \diamond A) \supset A>\diamond A$ has value 0 at some world $i$. Then $V(A, i)=1$ and $V(A>\diamond A, i)=0$. So $V(A, j)=1$ and $V(\diamond A, j)=0$ at some $j$ such that $j \in f(A, i)$. By Pseudo-centering, since $V(A, i)=1$, this implies $f(A, j)=\{j\}$. But this implies that $j R j$ and $V(A, j)=1$. It follows that $\diamond A$ is true at $j$ : contradiction. Secondly we consider $(A \wedge \diamond A) \supset \diamond A>A$. Suppose there is an $i$ such that $A \wedge \diamond A$ has value 1 in $i$ and $\diamond A>A$ value 0 at $i$. This means that there is a $j$ such that $j \in f(\diamond A, i), \diamond A$ has value 1 and $A$ value 0 at $j$. By Pseudo-centering, given that $V(\diamond A, i)=1$ and $j \in f(\diamond A, i)$, $f(\diamond A, j)=\{j\}$. Now $f$-symmetry states that, for every $i \in W$ and for every $A$ s.t. $V(A, i)=1$, if $j \in f(\diamond A, i)$, then $i \in f(\diamond A, j)$. But $f(\diamond A, j)$ is $\{j\}$. Thus $i$ belongs to $\{j\}$, which means that $i=j$. Since $A$ has value 0 at $j$, this means that at $i A$ has value 1 by hypothesis and also value 0 , which is impossible. So both Int and $\mathrm{X} 1$ are valid in all pseudo-centered and $f$-symmetrical $\mathbf{V}$ models. The expected result follows from standard induction on the length of proofs.

The proof that the systems VInt and VInt1 are distinct is as follows.

Proposition 2.4. VInt1 and VInt are non-equivalent systems.

Proof. It is enough to show that $\mathrm{X} 1$ is not a VInt-thesis. Let $M$ be a $\mathbf{V}$ model with the following consistent properties. $W=\{i, j\} ; V(A, i)=1$; $V(A, j)=0 ; i R i ; j \in f(\diamond A, i)$ and $i \in f(\diamond A, j)$. It is straightforward to see 
that $M$ is $f$-symmetrical, so it is a VInt-model. Furthermore $V(\diamond A, j)=$ 1 by the properties of $f$. Given that $V(\diamond A, j)=1$ and $V(A, j)=0$, so $V(\diamond A>A, i)=0$. Also, given that $i R i$ and $V(A, i)=1, V(\diamond A, i)=1$. So $V((A \wedge \diamond A) \supset((\diamond A>A), i)=0$ in $M$ and $V((A \wedge \diamond A) \supset(\diamond A><A), i)=0$ in $M$. Since all VInt-theses are valid in all $f$-symmetrical models, $\mathrm{X} 1$ is not a VInt-thesis.

The next result concerns the distinction of VInt1 and VInt1T.

\section{Proposition 2.5. VInt1 and VInt1 T are non-equivalent systems.}

Proof. It is enough to show that the axiom-schema $\mathrm{T}$ is underivable in VInt1. Let us consider a model $M$ with the following properties: $W=\{i\}, f(A, i)=$ $\emptyset, V(A, i)=1$. Since $f(A, i)=\emptyset$, due to the properties of selection functions, no $j$ exists such that $i R j$, so $\neg \diamond A$ has value 1 at $i$. Consequently $f(\diamond A, i)$ is also $\emptyset$ : otherwise $\diamond A$ would have value 1 at some $j$ s.t. $i R j$, so at $i$ (given that $W=\{i\})$, but this contradicts $V(\neg \diamond A, i)=1$. Since $f(\diamond A, i)=f(A, i)=\emptyset$, the conditions "if $k \in f(\diamond A, i)$, then $i \in f(\diamond A, k)$ " and "for every $j \in f(A, i)$, $f(A, j)=\{j\}$ ", jointly required for VInt1-models, are vacuously satisfied. Thus $M$ is a $\mathbf{V}$-model which is $f$-symmetric and pseudo-centered, so it is a VInt1-model. Since $A$ and $\neg \diamond A$ have both value 1 at $i, V(A \supset \diamond A, i)=0$. If $\mathrm{T}$ were a VInt1-thesis, $A \supset \diamond A$ would be also such, which is impossible.

VInt1 does not even have as a subsystem any system which contains the deontic axiom-schema $\mathrm{N}: \diamond T$.

Proposition 2.6. $\diamond \top$ is not a VInt1-thesis.

Proof. The same model $M$ built in Proposition 2.5 may be used to prove that $\square A$ and $\neg \diamond A$ have both value 1 in $M$, so the formula $\square A \supset \diamond A$ is falsified in $M$. Since $\square A \supset \diamond A$ is $\mathbf{V}$-equivalent to $\diamond \top, \diamond \top$ cannot be a VInt1-thesis.

The relation between the systems represented on the upper side of the diagram of Figure 1 and the ones on the right side are as follows. We call incomparable two systems $X$ and $Y$ when $X$ is not contained in $Y$ and $Y$ is not contained in $X$.

Proposition 2.7. VN is incomparable with VInt.

Organon F 26 (3) 2019: 427-445 
Proof. Since N is $\diamond T$, it cannot be derived in VInt, otherwise it would derivable in VInt1, which is excluded by Proposition 2.6. On the other hand, VInt is not a subsystem of VN. By Proposition 2.1 Int is not a V-theorem and the model $M$ built in the proof of Proposition 2.1 is a serial one, where seriality is the property by which, for every world $m$ in $M$ there is a world $n$ such that $m R n$. By a well-known result, $\mathrm{N}$ is valid in all serial models, so it is valid in $M$. So Int is falsified in a model which satisfies all $\mathbf{V N}$-theorems, so it is not a $\mathbf{V N}$-thesis.

Proposition 2.8. $\boldsymbol{V W}$ is incomparable with VInt1.

Proof. From the axiom CMP, i.e. $A>B \supset(A \supset B)$, it follows by instantiation $\neg A>A \supset(\neg A \supset A)$, so $\square A \supset A$, i.e. the schema called T. But we already proved that $\mathrm{T}$ is underivable in VInt1, so $\mathbf{V W}$ is not a subsystem of VInt1. On the other hand Int, i.e., $(A \wedge B) \supset \diamond A>\diamond B$, is underivable in $\mathbf{V W}$. A $\mathbf{V W}$-countermodel $M$ to Int may be built as follows. $W=\{i, j, k\}, V(A, i)=V(A, k)=V(B, i)=1$ and $V(B, j)=V(B, k)=0$; $j \in f(\diamond A, i) ; j R k$. Furthermore $i \in f(A, i), i \in f(B, i), j \in f(\neg B, j)$, $k \in f(A, k), k \in f(\neg B, k)$. From this definition it follows that $M$ is a VWmodel since it has the property of Weak-Centering (i.e. that, for every $A$ and every $h$, if $V(A, h)=1, h \in f(A, h))$. It also follows from the definition that $i R i, j R j, k R k$, i.e. total reflexivity. Since $j R k$ and $V(A, k)=1$, $V(\diamond A, j)=1$. But $V(\diamond B, j)=0$ since $j R k, j R j$ and $B$ has value 0 in both $k$ and $j$. Given that $j \in f(\diamond A, i)$, it turns out that $A \wedge B$ has value 1 while $\diamond A>\diamond B$ has value 0 at $i$. So $M$ is a $\mathbf{V W}$-countermodel for Int and Int cannot be a $\mathbf{V W}$-theorem.

\section{The deductive equivalence between VInt1T and VC}

We now may prove the deductive equivalence between VInt1T and VC. For the desired result we need the following two Lemmas.

Lemma 3.1. $R B: \checkmark A \supset(A>B \supset A \ni B)$ (Restricted Boethius) and PCI: $\square \neg A \supset A>B$ (Paradox of Conditional Implication) are $\boldsymbol{V}$-theorems.

Proof. Assume by Reductio the negation of (RB), i.e. $\diamond A \wedge A>B \wedge A>\neg B$. This implies, in $\mathbf{V}, \diamond A \wedge(A>(B \wedge \neg B)), \diamond A \wedge(A>\perp)$, so $\diamond A \wedge \neg \vee A$ : contradiction. Then RB is a $\mathbf{V}$-theorem. 
(PCI) By rule RCK, $\vdash_{\text {PC }} \neg A \supset(A \supset \perp)$ implies $\vdash A>\neg A \supset A>(A \supset$ $\perp)$. So again by RCK, by Id and by Transitivity of $\supset, \vdash A>\neg A \supset A>\perp$. $\vdash_{\text {PC }} \perp \supset B$ implies, again by RCK, $\vdash A>\perp \supset A>B$. So, by Transitivity of $\supset$ and by Def $\square, \square \neg A \supset A>B$.

Proposition 3.2. In VInt1T X1 is equivalent to $X 1^{\circ}: A \supset \diamond A><A$.

Proof. A theorem of VInt1T is $A \supset \diamond A$, so $(A \wedge \diamond A) \equiv A$. Then X1, i.e. $(A \wedge \diamond A) \supset(\diamond A><A)$, by Replacement of Material Equivalents turns out to be equivalent to $A \supset \diamond A><A$.

Proposition 3.3. For any formula $A, A$ is a VInt1T-thesis iff $A$ is $\boldsymbol{V} \boldsymbol{C}$ thesis.

Proof. $(\Longrightarrow)$ All the three wffs which are axioms schemata of VInt1T are theorems of VC. In fact, (a) T: $\square A \supset A$ and its variant $A \supset \diamond A$ follow from CMP. (b) An instantiation of CS is $(\diamond A \wedge \diamond B) \supset \diamond A>\diamond B$ but, given the KT-theorem $(A \wedge B) \supset(\diamond A \wedge \diamond B)$, by transitivity of $\supset$ we reach $(A \wedge B) \supset$ $\diamond A>\diamond B$, i.e. Int. (c) An instance of CS is $(A \wedge \diamond A) \supset \diamond A>A$. Since $\vdash A>\diamond A$ follows from $\vdash A \supset \diamond A$ by RCE, it is straightforward to derive $(A \wedge \diamond A) \supset \diamond A><A$, i.e. X1.

$(\Longleftarrow)$ The following is a syntactical proof of CS in VInt1T.

1. $A \supset \diamond A><A$

$\mathrm{X} 1^{\circ}$

2. $B \supset \diamond B><B$

$\mathrm{X} 1^{\circ}$

3. $(A \wedge B) \supset \diamond A><A$

$(1), \vdash(A \wedge B) \supset A, \mathbf{P C}$

4. $(A \wedge B) \supset \diamond B>B$

(2), PC

5. $A>B \supset(A \ni C \supset(A \wedge C)>B)$

$\mathrm{CV}, \mathbf{P C}$

6. $\diamond B>B \supset(\diamond B \ni \diamond A \supset(\diamond B \wedge \diamond A)>B)$

7. $(A \wedge B) \supset(\diamond B \ni \diamond A \supset(\diamond A \wedge \diamond B)>B)$

(5), $\diamond B$ for $A, \diamond A$ for $C$

8. $\diamond A \supset(A><B \supset A \ni B)$

(4), (6), PC

9. $\diamond \diamond A \supset(\diamond A><\diamond B \supset \diamond A \ni \diamond B)$

$\mathrm{RB}, \mathrm{PC}$

10. $(A \wedge B) \supset \diamond A><(\diamond A \wedge \diamond B)$

11. $\diamond \diamond A \supset((A \wedge B) \supset \diamond A \ni \diamond B)$

12. $\diamond \diamond B \supset((A \wedge B) \supset \diamond B \ni \diamond A)$

13. $\diamond \diamond B \supset((A \wedge B) \supset(\diamond A \wedge \diamond B)>B)$

$(8), \diamond A$ for $A, \diamond B$ for $B$
$\diamond A \wedge \diamond B)) \equiv \diamond A><\diamond B$

14. $\diamond \diamond B \supset((A \wedge B) \supset \diamond A>B)$

15. $\diamond \diamond B \supset((A \wedge B) \supset A>B)$

16. $\neg \diamond \diamond B \supset((A \wedge B) \supset C)$

17. $\neg \diamond \diamond B \supset((A \wedge B) \supset A>B)$ Int, $\vdash_{\mathbf{V}}(\diamond A><(\diamond A \wedge \diamond B)) \equiv \diamond A><\diamond B$
$(9)$, Int, $\mathbf{P C}$

18. $(A \wedge B) \supset A>B$

(11), $B$ for $A$ and $A$ for $B$, PC

(12), (7), PC

(13), (10), CSO, PC (14), (3), CSO $\vdash_{\mathbf{K T}} \square \square \neg B \supset \neg B, \vdash_{\mathbf{P C}} \neg B \supset((A \wedge B) \supset C)$

(16), $A>B$ for $C$

(17), (15), PC

Organon F 26 (3) 2019: 427-445 
It remains to be proved that CMP, i.e. $A>B \supset(A \supset B)$, is a theorem of VInt1T. From RB: $\diamond A \supset(A>B \supset A \ni B)$ (See Lemma 3.1) and CS: $(A \wedge B) \supset A>B$ one obtains $\diamond A \supset((A \wedge B) \supset A \ni B)$, so $\diamond A \supset(A>\neg B \supset(A \supset \neg B))$ and obviously

(*) $\diamond A \supset(A>B \supset(A \supset B))$.

But $\neg \vee A \supset(\neg A \vee B)$ is a $\mathbf{K T}$-thesis, so a fortiori:

$$
(* *) \quad \neg \diamond A \supset(A>B \supset(A \supset B))
$$

is such. Then by $(* *)$ and $(*) A>B \supset(A \supset B)$, i.e. CMP, follows in a straightforward way.

As proved by Lewis, VC is sound and complete w.r.t. the class of all centered models. A simple semantic remark helps to understand the equivalence between the two systems VC and VInt1T.

Proposition 3.4. Any $\boldsymbol{V}$-model $M$ is centered if and only if $M$ is pseudocentered, reflexive and $f$-symmetric.

Proof. (a) It is easy to see that every centered $\mathbf{V}$-model $M$ is a fortiori pseudocentered, reflexive and $f$-symmetric. Reflexivity follows obviously by Centering. Furthermore, if $V(A, i)=1$, by Reflexivity $V(\diamond A, i)=1$, so by Centering $f(\diamond A, i)=\{i\}$ : hence $f(A, i)=f(\diamond A, i)$, given that both are identical to $\{i\}$. Hence if $j \in f(A, i), j=i$.

The two properties $f S$ and $P s C$ follow in the following way.

$(f S)$ For every $i$ in $W$ such that $V(A, i)=1$, if $j \in f(\diamond A, i)$, by the proved identity $f(A, i)=f(\diamond A, i), j \in f(A, i)$; then by the identity $j=i$, $i \in f(\diamond A, j)$. So, supposing that $V(A, i)=1$, if $j \in f(\diamond A, i), i \in f(\diamond A, j)$.

$(P s C)$ For every $i$ in $W$, if $V(A, i)=1$ then, being proved by the Centering property that $f(A, i)=\{i\}$, it follows that $i=j$ for every $j$ s.t. $j \in f(A, i)$. So, if $V(A, i)=1$, by substitution of $j$ to $i f(A, j)=\{j\}$, which is the property of Pseudo-centering.

b) It may be proved that every pseudo-centered $f$-symmetric reflexive model is a centered model. Suppose $V(A, i)=1$. Then by Reflexivity $V(\diamond A, i)=1$. One can prove that $f(\diamond A, i)$ is not $\emptyset: f(\diamond A, i)=\emptyset$ in fact 
would imply the truth at $i$ of $\diamond A>C$ for every $C$, so the truth of $\diamond A>\neg \nabla A$, of $\square \neg \diamond A$ (by Def $>$ ) and of $\square \square \neg A$, so by reflexivity the truth of $\neg A$ at $i$, which is the negation of the hypothesis $V(A, i)=1$. Then there is a $j \in f(\diamond A, i)$, and in such $j \diamond A$ has value 1 . Now Pseudo-centering holds for every wff, so also for $\diamond A$. Hence, for every $i$ in $W$, and for every $A$ such that $V(\diamond A, i)=1$ we have, for every $j$ in $f(\diamond A, i), f(\diamond A, j)=\{j\}$ and we know that there is at least one $j$ of this kind. And, given that $j \in f(\diamond A, i)$ implies $i \in f(\diamond A, j)$ by $f$-symmetry, $i \in\{j\}$ and $j=i$. Since $f(\diamond A, j)=\{j\}$, by substitution of identicals $f(\diamond A, i)=\{i\}$. Since $A$ is true at $i$, it is also true at every world of $f(\diamond A, i)$, so $\diamond A>A$ is true at $i$. Given that $A>\diamond A$ is true at $i$ and at all the possible worlds of any reflexive model, then $\diamond A><A$ has value 1 at $i$. This means, by property b) of the function $f$ (see section 1 ), that $f(A, i)=f(\diamond A, i)$. Given that $f(\diamond A, i)=\{i\}$, we conclude that, if $V(A, i)=1, f(A, i)=\{i\}$, which expresses the condition of Centering.

\section{CS as a source of partial trivialization of modal operators}

The two basic laws of central systems of classical conditional logics, CMP and CS, have been object of criticism by various scholars. Conditional Modus Ponens has been criticized by (McGee 1985) and is not a theorem of the system $\AA$ in (Aqvist 1973). The reason is that if $A>B$ is interpreted by something as $* A-3 B$ (where $*$ is the so called "circumstantial operator" indicating the conjunction of $A$ with a tacitly understood antecedent), an acceptable version of conditional Modus Ponens would be not $A>B \supset(A \supset B)$ but $A>B \supset(* A \supset B)$ : but the latter formula is inexpressable in a language having $>$ as the only primitive. As far as CS is concerned, suffice it to mention (von Kutschera 1974) and (Gundersen 2004) just to quote only two papers in which the conditional systems under analysis omit CS. The principle has been criticized also in (Bennett 1974) and (Bigelow 1976). What we want to stress in this paper is that the implausibility of CS, and more generally of system VC, is due to the fact that CS is a source of a partial or full trivialization of some non-truthfunctional operators definable in terms of $>$. As observed at the beginning, if the necessity operator is defined as $\square A=_{d f} \top>A$, the collapse-formula Triv $\square$ : $\square A \equiv A$ is derivable in VC but not in the weaker 
system, being a consequence of the introduction of CS.

It is of some interest to remark that Def $\square$ and Def $\square$ allow for a different axiomatization of $\mathbf{V C}$ consisting in two simple axiom-schemata with only one metavariable. Let us call $\mathrm{V} \square \square$ the system which is obtained by extending the axiomatic basis of $\mathbf{V}$ with the definitions of $\square$ and $\square$ and the following two schemata:

$$
\begin{array}{ll}
\mathrm{Ban} \bullet & A \supset \square A \\
\mathrm{~T} & \square A \supset A
\end{array}
$$

Then we may prove the following metatheorem.

Proposition 4.1. $\boldsymbol{V} \sqcup \square$ and $\boldsymbol{V} \boldsymbol{C}$ are deductively equivalent systems.

Proof. The rules of the two systems and the axioms of $\mathbf{V}$ are common to $\mathbf{V} \square \square$ and VC, so it is enough to do these two steps.

$(\Longrightarrow)$ We prove that CS and CMP are derivable in $\mathrm{V} \square \square$. As for CS:

1. $A \supset \top>A$

2. $A>\mathrm{T}$

Ban $\square$, Def $\square$

PC, RCE

3. $A \supset \top><A$

(1), (2), PC

4. $B \supset \top><B$

(3), $B$ for $A$

5. $(A \wedge B) \supset(T><B \wedge T><A)$

6. $\top><A \supset(\top>B \equiv A>B)$

7. $(\top><A \wedge \top><B) \supset A>B$

8. $(A \wedge B) \supset A>B$

(3),(4), Theorema Praeclarum CSO, T for $A, A$ for $B, B$ for $C$ (6), PC, $\vdash B>\top((2), B$ for $A)$

(5), (7), PC

As for CMP: The same proof as for the wffs $(*)$ and $(* *)$ used in the proof of Proposition 3.3, which are steps to derive CMP from T and CS.

$(\Longleftarrow)$ We have already shown that the schema Ban is derivable in VC, while $\mathrm{T}$ is a trivial consequence of CMP.

Proposition 4.1 implies that $\mathrm{Ban} \sqcup: A \supset \square A$ and $\operatorname{Triv} \odot: A \equiv \square A$ are equivalent in $\mathrm{V} \square \square$, since $\square A \supset A$ easily follows from CMP.

Notice that in systems having -3 as primitive (see, for instance, S1-S5 introduced in Lewis and Langford 1932) $\square A$ may be indifferently defined as $\neg A \neg A$ and $(A \vee \neg A) \neg A$. However, in conditional logic the distinction between $\square A$ and $\square A$ depends, via Def $\square$ and Def $\square$, on the distinction between $(A \vee \neg A)>A$ e $\neg A>A$. The reason why such two formulas cannot be logically equivalent in classical conditional logics is that there is no room in them for the so-called Simplification of Disjunctive Antecedents 
$\mathrm{SDA} \quad(A \vee B)>C \supset B>C$.

Thanks to SDA, in fact, $(A \vee \neg A)>A$ (i.e., $\square A$ ) would imply $\neg A>A$ (i.e., $\square A$ ), while the converse implication follows from MOD. But the failure of SDA is a weak point of classical conditional logic. (Nute 1980, $40 \mathrm{ff}$.) stresses with a different argument the incompatibility between SDA and CS, and introduces a class of non-standard conditional logics in which the first, but not the latter theorem, is allowed. As is well-known, Lewis has defended CS by claiming that conditional logic is interested in truth conditions and not in relevance conditions between the clauses. The problem of the "disconnection" of the clauses is traditionally seen as a weak point of the material conditional. This helps to understand a second kind of trivialization yielded by CS in conjunction with CMP: in fact, their combination leads one to prove the equivalence between classical conditionals with a true antecedent and material conditionals with a true antecedent.

A conditional which is stated in conjunction with the truth of its antecedent may be read as a "since" conditional or, as (Goodman 1947) says, a factual conditional. Thanks to CMP, the factual conditional $A \wedge A>B$ entails $(A \wedge B) \wedge A>B$ and vice versa. Thanks to CS, $A \wedge B$ entails $A>B$, so $(A \wedge B) \wedge A>B$ turns out to be equivalent to $A \wedge B$. So we reach soon the equivalence

(о) $A \wedge B \equiv A \wedge(A>B)$

(o) states that any factual conditional collapses on the conjunction of its clauses. Furthermore, given that $A \wedge B$ is $\mathbf{P C}$-equivalent to $A \wedge(A \supset B)$, we have the collapse of factual material conditionals on factual classical conditionals, that is a special case of trivialization

$$
\text { (॰०) } A \wedge(A \supset B) \equiv A \wedge(A>B) \text {. }
$$

In the present paper attention has been given to a third kind of trivializiation. Let us recall that

$\mathrm{X} 1^{\circ} \quad A \supset \diamond A><A$

Organon F 26 (3) 2019: 427-445 
follows from $\mathrm{CS}$ in the system $\mathbf{V C}$ and that it entails $\mathrm{CS}$ in the system VInt1T. X $1^{\circ}$ asserts that if $A$ is true, $A$ is $a$-equivalent with its own possibility. Let us read again $A \wedge(A>C)$ as "Since $A, C$ " and let us suppose that $A \wedge(A>C)$ is true (e. g. "Since it rained, streets are wet"). $A \wedge(A>C)$ implies $A$ and, by $\mathrm{X} 1^{\circ}, A$ implies $\diamond A><A$. Thanks to CSO, then, from $A>C$ we derive $\nabla A>C$. And, given that we have at our disposal also the law $A \supset \diamond A$, we conclude that the conjunction $A \wedge(A>C)$ entails the conjunction $\diamond A \wedge \diamond(A>C)$. More formally, we derive the following formula:

$$
(\circ \circ \circ) \quad(A \wedge(A>C)) \supset(\diamond A \wedge(\diamond A>C)) \text {. }
$$

Following the proposed interpretation of $A$ and $C$, then the consequent of ( $\circ \circ)$ says "streets are wet since it is logically possible that it rained", i.e. is something which is intuitively false, while the antecedent $A \wedge(A>C)$ appears to be intuitively true. One might be willing to remark that this result is simply an apparent anomaly, since the background conception of conditionals which inspired Stalnaker-Lewis systems is divisionist. In other words the background theory claims that there are two kinds of conditionals (roughly: the indicative and the counterfactual) and the truth-conditions for the indicative conditional are the same as the ones for the material conditional. However, the preceding remark should face the content of the following metatheorem:

Proposition 4.2. In $\boldsymbol{V} \boldsymbol{C}$ the thesis $X 1^{\circ}: A \supset \diamond A><A$ is logically equivalent to $X 1 \vee: \diamond A><A \vee \diamond \neg A><\neg A .^{4}$

Proof. The proof consists in proving the two sides of the equivalence which we call here (ia) and (ib). Let us recall that $\mathbf{V C}$ contains $\mathbf{V W}$, hence the $\square$-logic KT and also the theorems $A \supset \diamond A$ and $A>\diamond A$.

\footnotetext{
${ }^{4} \diamond A><A \vee \diamond \neg A><\neg A$ should not be confused with $\square A><A \vee \square \neg A><\neg A$. One may prove that $\square A><A \vee \square \neg A><\neg A$ is equivalent to $\neg A \supset \square A><A$, and the latter formula may be proved to be equivalent to the two-metavariable wff $A \supset(B>(A>B)$ ), which is not VC-valid (the proofs will not be given here).
} 
(ia)

1. $\vdash \diamond A><A \vee \diamond \neg A><\neg A$

2. $(\diamond A \wedge \neg A) \supset \neg(\diamond A>A)$

3. $(\diamond A \wedge \neg A) \supset \neg(\diamond A><A)$

4. $(\diamond A \wedge \neg A) \supset \diamond \neg A><\neg A$

5. $\neg \diamond A \supset \diamond \neg A><\neg A$

6. $(\neg \diamond A \wedge \neg A) \supset \diamond \neg A><\neg A$

7. $\neg A \supset \diamond \neg A><\neg A$

8. $A \supset \diamond A><A$

$\mathrm{X} 1 \mathrm{~V}$

CMP, PC, $\diamond A$ for $A, A$ for $B$

(2), Def $><, \vdash A>\diamond A$

(3), (1), PC

MOD, $\diamond \neg A$ for $B, \neg A$ for $A, \vdash \neg A>\diamond \neg A$

(5), PC

$\vdash_{\mathbf{K T}}((\neg A \wedge \diamond \neg A) \vee(\neg A \wedge \diamond A)) \equiv \neg A$,

(4), (6), PC

(7), $\neg A$ for $A, \mathbf{P C}$

(ib)

1. $\vdash A \supset \diamond A><A$

$\mathrm{X} 1^{\circ}$

2. $\neg A \supset \diamond \neg A><\neg A$

(1), $\neg A$ for $A$

3. $((A \supset B) \wedge(C \supset D)) \supset((A \vee C) \supset(B \vee D))$

$\mathrm{PC}$

4. $(A \vee \neg A) \supset(\diamond A><A \vee \diamond \neg A><\neg A)$

(1), (2), (3) ( $\neg A$ for $C, \diamond A><A$

5. $\diamond A><A \vee \diamond \neg A><\neg A$ for $B, \diamond \neg A><\neg A$ for $D$ ), MP $(4), \vdash \top, \mathrm{MP}$

As a consequence of Proposition 4.2, an axiom system which is equivalent to VInt1T (so to VC and to $\mathbf{V} \bullet \square$ ) is the following, which we call here VInt1 T $^{o}$ :

Int $\quad(A \wedge B) \supset \diamond A>\diamond B$

$\mathrm{X} 1 \vee \diamond A><A \vee \diamond \neg A><\neg A$;

$\mathrm{T} \quad \square A \supset A$.

Now the disjunction $\mathrm{X} 1 \mathrm{~V}$ implies that at least one of the two statements $A$ and $\neg A$ is $a$-equivalent with its own possibility. This statement does not make any assertion containing factual conditionals. Thanks to X1V we are able to perform in $\mathbf{V C}$ the following proof. In order to simplify notation, we recall that $\diamond \neg A \wedge \diamond A$ means that $A$ is contingent and is symbolically represented usually by $\nabla A$.

Organon F 26 (3) 2019: 427-445 
1. $(A>B \wedge A \ni C) \supset(A \wedge C)>B$

2. $\diamond \neg A>\neg A \supset((\diamond \neg A \ni \diamond A) \supset(\diamond \neg A \wedge \diamond A)>\neg A)$

3. $\diamond A><A \vee((\diamond \neg A \ni \diamond A) \supset(\diamond \neg A \wedge \diamond A)>\neg A))$

4. $((\diamond \neg A \ni \diamond A) \wedge(\diamond \neg A \wedge \diamond A) \ni A) \supset(\diamond A><A)$

5. $((\diamond \neg A \ni \diamond A) \wedge(\nabla A \ni A)) \supset \diamond A><A$

6. $(\diamond \neg A \wedge \diamond A) \supset(\diamond \neg A \ni \diamond A)$

7. $(\nabla A \wedge(\nabla A \ni A)) \supset \diamond A><A$
CV

(1) $\diamond \neg A$ for $A, \diamond A$ for $C$, $\neg A$ for $B, \mathbf{P C}$ $\mathrm{X} 1 \mathrm{~V},(2), \mathrm{MP}$

(3), $\vdash A \vee B \equiv \neg B \supset A$, Def $\ni$

(4), Def $\nabla$

CMP, PC

(6), (5), PC, Def $\nabla$

How can we read (7)? It asserts the if $A$ is contingent and its contingency is cotenable with $A$, then $A$ and $\diamond A$ are $a$-equivalent. But most actual and possible facts of real life have the property stated in the antecedent of this implication. So, given that raining is a contingent fact and that such property does not exclude rain, raining is a-equivalent to its own possibility. Given the mentioned premises, as a result of $a$-equivalence we may then replace $A$ and $\diamond A$ in the antecedents of any appropriate conditional. In many cases this replacement however leads to bizarre conclusions. For instance, we can imagine a scenario in which yesterday it did not rain $(\neg A)$ but rain was clearly a possibility $(\diamond A)$. Now let us consider the true counterfactual:

(i) if it had rained, streets would have been wet $(A>W)$

and from (i) and the other premises we conclude, exploiting replacement of $a$-equivalents

(ii) if rain had been a possibility, streets would have been wet $(\diamond A>W)$

(ii) is not only false but, thanks to the true premise $\diamond A$, it allows to go further: we may conclude by CMP $\diamond A \supset W$ and by standard Modus Ponens, from $\checkmark A$, that also $W$ is true, i.e. that streets were wet yesterday: very strange conclusion since among our premises there is the fact that yesterday it did not rain.

\section{Acknowledgements}

The author wishes to thank the editors and an anonymous referee for useful corrections to the first draft of the paper, which was quite different from the actual version. 


\section{References}

Bennett, Jonathan. 1974. "Counterfactuals and Possible Worlds." Canadian Journal of Philosophy 4(2): 381-402. https://doi.org/10.1080/00455091.1974.10716947

Bigelow, John C. 1976. "If-then Meets the Possible Worlds." Philosophica 6(2): 215-236. https://doi.org/10.1007/BF02379924

Chellas, Brian. 1975. "Basic Conditional Logic." Journal of Philosophical Logic 4(2): 133-154. https://doi.org/10.1007/BF00693270

Goodman, Nelson. 1947. "The Problem of Counterfactual Conditionals." Journal of Philosophy 44(5):113-128. https://doi.org/10.2307/2019988

Gundersen, Lars B. 2004. "Outline of a New Semantics for Counterfactuals." Pacific Philosophical Quarterly 85(1): 1-20. https://doi.org/10.1111/j.1468-0114.2004.00184.x

Lewis, Clarence Irving and Langford, Cooper Harold. 1932. Symbolic Logic. New York: The Century Company.

Lewis, David K.. 1971. "Completeness and Decidability of Three Logics of Counterfactuals." Theoria 37(1): 74-85. https://doi.org/10.1111/j.1755-2567.1971.tb00061.x

Lewis, David K. 1973. Counterfactuals. Oxford: Blackwell.

McGee, Vann. 1985. "A Counterexample to Modus Ponens." Journal of Philosophy 82(9): 462-571. https://doi.org/10.2307/2026276

Nute, Donald. 1980. Topics in Conditional Logic. Dordrecht: Reidel.

Stalnaker, Robert. 1968. "A Theory of Conditionals." In Studies in Logical Theory, edited by N. Rescher, 98-112. Oxford: Blackwell.

von Kutschera, Franz. 1974. "Indicative Conditionals." Theoretical Linguistics 1 (1-3): 257-269. https://doi.org/10.1515/thli.1974.1.1-3.257

Åqvist, Lennart. 1973. "Modal Logic with Subjunctive Conditionals and Dispositional Predicates." Journal of Philosophical Logic 2(1): 1-76.

https://doi.org/10.1007/BF02115609

Organon F 26 (3) 2019: 427-445 